\title{
Treinamento Cognitivo de Atenção e Memória de Universitários Com Jogos Eletrônicos
}

\author{
Sabrina Martins Barroso ${ }^{1}$ \\ Fernanda Esteves Pereira ${ }^{2}$ \\ Dennis Gabiatti Lopes ${ }^{3}$ \\ Juliana Ruiz Machado ${ }^{4}$ \\ José Humberto Curtiço Júnior ${ }^{5}$
${ }^{1}$ Universidade Federal do Triângulo Mineiro, MG, Brasil http://orcid.org/0000-0003-1759-9681
${ }^{2}$ Universidade Federal do Triângulo Mineiro, MG, Brasil
https://orcid.org/0000-0002-9554-3301
${ }^{3}$ Universidade Federal do Triângulo Mineiro, MG, Brasil
$\therefore$ http://orcid.org/0000-0003-3721-9922
${ }^{4}$ Universidade Federal do Triângulo Mineiro, MG, Brasil
https://orcid.org/0000-0002-0895-5253
${ }^{5}$ Universidade Federal do Triângulo Mineiro, MG, Brasil \\ http://orcid.org/0000-0001-8795-4795
}

\begin{abstract}
Resumo
Estudos têm mostrado o potencial dos videogames para treinar habilidades cognitivas. O presente estudo verificou a eficácia de um treinamento com jogos eletrônicos para melhoria das funções cognitivas de atenção e memória de estudantes universitários. Participaram da pesquisa 25 universitários, com idade entre 18 e 29 anos, de ambos os sexos. Utilizou-se os testes de Trilhas, Nomeação de Boston, Lista de Palavras, Subteste dígitos das Escalas Wechsler de Avaliação de Inteligência Adulta, Teste do Desenho do Relógio, Fluência Verbal: Animais e Questionário de Queixa Subjetiva de Memória. O treinamento com jogos eletrônicos utilizou jogos comerciais, escolhidos pela equipe de pesquisa. O treino durou entre 5 e 16 semanas, com tempo progressivo de treinamento variando de 20 a 60 minutos. Os participantes eram equivalentes nas funções cognitivas no pré-teste. Após a intervenção, foram observadas diferenças na memória imediata, memória tardia, reconhecimento, recordação de dígitos em ordem inversa, atenção e nomeação. Os resultados indicam que os jogos eletrônicos podem ser ferramentas úteis para treinar as habilidades cognitivas, mas como a amostra é pequena, estudos futuros são necessários para ampliar os conhecimentos na área.
\end{abstract}

Palavras-chave: jogos de vídeo, atenção, memória, jovem adulto.

\section{Cognitive Training of Attention and Memory of College Students With Eletronic Games}

Abstract
Previous studies have shown the potential of video games to train cognitive skills. The present study verified the effectiveness of a training
with electronic games to improve the attention and memory skills of undergraduated students. Twenty-five students aged between 18 and 29 ,


of both genders participated the study. It was used Track tests, Boston Naming, Word List, Subtest digits of the Wechsler Adult Intelligence Assessment Scales, Clock Drawing Test, Verbal Fluency: Animals, and Subjective Memory Complaint Questionnaire. Electronic game training used commercial games, chosen by the research team. The training lasted between 5 and 16 weeks, with progressive training time ranging from 10 to 60 minutes. The participants had equivalent cognitive functions pre-test, but differences in immediate memory, late memory, recognition, recall of digits in reverse order, attention, and naming were observed after the intervention. The results indicate that electronic games may be useful tools for training cognitive skills, but because the sample is small, future studies are needed to expand knowledge in the field. Keywords: video game, attention, memory, young adult.

\section{Formación Cognitiva de Atención y Memoria De Miembros Universitarios con Juegos Electrónicos}

\section{Resumen}

Los estudios han demostrado el potencial de los videojuegos para entrenar habilidades cognitivas. El presente estudio verificó la efectividad del entrenamiento con juegos electrónicos para mejorar las funciones cognitivas de atención y memoria de los estudiantes universitarios. Veinticinco estudiantes universitarios, con edades comprendidas entre 18 y 29 años, de ambos sexos participaron en la investigación. Se utilizaron senderos, nombres de Boston, lista de palabras, dígitos de subprueba de la escala de clasificación de inteligencia para adultos de Wechsler, prueba de dibujo de reloj, fluidez verbal: animales y cuestionario de queja de memoria subjetiva. La capacitación con juegos electrónicos utilizó juegos comerciales, elegidos por el equipo de investigación. El entrenamiento duró entre 5 y 16 semanas, con un tiempo de entrenamiento progresivo de 20 a 60 minutos. Los participantes fueron equivalentes en funciones cognitivas en la prueba previa, después de que las diferencias de intervención se observaron en la memoria inmediata, memoria tardía, reconocimiento, memoria inversa de dígitos, atención y nomenclatura. Los resultados indican que los juegos electrónicos pueden ser herramientas útiles para entrenar habilidades cognitivas, pero como el tamaño de la muestra es pequeño, se necesitan estudios futuros para ampliar el conocimiento en el area.

Palabras claves: videojuegos, atención, memoria, adultos jóvenes.

Os jogos eletrônicos são dispositivos computacionais desenvolvidos prioritariamente para entretenimento, que geram grande polêmica sobre o seu impacto no comportamento social dos jogadores (Baum \& Maraschin, 2017) e frequentemente somente aspectos negativos dos jogos, tais como agressividade e vício são investigados (Gnambs \& Appel, 2017). Fora dos aspectos controversos, os jogos têm mostrado impacto positivo como forma de treinamento cognitivo (Steenbergen, Sellaro, Stock, Beste, \& Colzato, 2015; Kristjánsson, 2013; Chiappe, Conger, Liao, Caldwell, \& Vu, 2013; Mishra, Bavelier, \& Gazzaley, 2012; Bavelier, Achtman, Mani, \& Focker, 2012; Nouchi et al., 2013; Boot, Kramer, Simons, Fabiani, $\&$ Gratton, 2008), treinamento físico (Severini et al., 2017), melhoria de condições emocionais (Francis, Comello, \& Marshall, 2016), mudanças de hábitos alimentares em crianças e adolescentes (Thompson et al., 2015) e reabilitação de substâncias (Cutter et al., 2014).

Baum e Maraschin (2017) apontam que a experiência como jogador possibilita uma vivência distinta e fomenta a chance de aprendizado de diversas habilidades. Tais habilidades são tanto cognitivas quanto sociais, dependendo do tipo de jogo experimentado. Oliveira (2007) indica que quando o cérebro de um jogador está direcionado para a resolução de um problema, inicia-se um processo de busca e seleção de estratégias, pelas quais atenção e memória trabalham em conjunto para se chegar a uma solução, o que possibilita aprendizado. Outros estudos mostram melhora das habilidades dos jogadores na atenção e redução do tempo de reação para realização de tarefas (Mishra et al., 2013; Kristjánsson, 2013; Li, Polat, Scalzo \& Bavelier, 2010; Dye \& Bavelier, 2010) e implemento na memória (Colzato, van den Wildenberg, Zmigrod, \& Hommel, 2012; Boot et al., 2010; Thorell et al., 2009; VanMuijden et al., 2012). Além disso, há também estudos que mostraram o aprimoramento da capacidade de realizar mais de uma tarefa ao mesmo tempo (Chiappe et al., 2013; Steenbergen et al., 2015) e na capacidade para tomada de decisões executivas (Boot, Kramer, Simons, Fabiani, \& Gratton, 2008) entre jogadores. Enquanto outros mostraram melhor desempenho visual dos jogadores em tarefas que demandam 
localização espacial, identificação rápida de alvos, rastreamento de mais de um objeto e maior controle em tarefas que apresentam alta carga de informações (Green \& Bavelier, 2006; Chisholm et at., 2010).

Alguns estudos apontam, até mesmo mudanças estruturais e funcionais no cérebro dos jogadores (Bavelier, Achtman, \& Föcker, 2012; Klingberg, 2010; Neubauer, Bergner, \& Schatz, 2010; Scholz, Klein, Behrens, \& Johansen-Berg, 2009), em especial no lobo frontal e parietal dos jogadores. Draganski et al. (2010) afirmam que praticar e aprender novas habilidades pode influenciar nas mudanças estruturais e funcionais do cérebro. Acredita-se que tais alterações ocorrem como forma do cérebro se adaptar às demandas de especialização geradas pelo hábito de jogar e às demandas das tarefas dos jogos, contribuindo para a melhoria no desempenho dos jogadores quanto às habilidades cognitivas (Bavelier, Achtman, \& Föcker, 2012; Klingberg, 2010). Assim, os jogos poderiam fornecer os estímulos necessários para a aprendizagem e a ativação de diferentes regiões cerebrais, sensibilizando o cérebro para captar pistas e encontrar uma solução mais rápida.

A prática e a repetição de tarefas auxiliam na aquisição de habilidades, incluindo as cognitivas. Os jogos contêm cenários, músicas e efeitos pensados para manter o interesse do jogador, que são aspectos que podem contribuir para a motivação para a realização da repetição de atividades (Gnambs \& Appel, 2017). Paralelamente, os videogames tendem a mostrar algum tipo de medida de desempenho (ex: vidas, pontos), que permitem mensurar o desempenho dos jogadores, o que é um aspecto desejável em treinamentos cognitivos (Aranha, 2006). Ao avaliar a produção sobre uso de jogos eletrônicos em programas de treinamento cognitivo percebe-se uma escassez de estudos brasileiros sistematizados sobre esse tema. Um dos poucos trabalhos foi desenvolvido por Alves e Carvalho (2010). Nesse estudo, os autores avaliaram a atenção de pessoas que não jogavam videogames, implementaram um treinamento utilizando jogos eletrônicos e reavaliaram os participantes. Observou-se que a intervenção foi eficaz para melhorar o desempenho dos participantes na capacidade de atenção sustentada. Considerando o possível potencial dos jogos como ferramenta de treinamento cognitivo, o objetivo desse estudo foi verificar a eficácia de um treinamento com jogos eletrônicos para melhoria das funções cognitivas de atenção e memória de estudantes universitários.

\section{Método}

\section{Participantes}

Participaram da pesquisa 25 estudantes universitários da Universidade Federal do Triângulo Mineiro (UFTM), com idade entre 18 e 29 anos (=21,08; D. P. $\pm 2,63)$, sendo 18 do sexo feminino (72\%) e sete do sexo masculino (28\%). A amostra foi selecionada por conveniência, entre estudantes regularmente matriculados na UFTM que indicaram não jogar videogame previamente.

\section{Instrumentos}

Questionário de Queixa Subjetiva de Memória. Desenvolvido por Tobiansky, Blizard, Livingston e Mann (1995), avalia as queixas dos respondentes por meio de nove perguntas com alternativas de resposta em escala likert. Pelo total de pontos chega-se às categorias "sem queixa" (soma dos pontos das respostas entre zero e dois pontos), queixa leve (três e cinco pontos), queixa moderada (seis e oito pontos) e severa (nove e doze pontos).

Teste de Atenção de Trilhas (Forma B). Desenvolvido por Montiel e Capovilla (2009), inicialmente pensado para avaliação infantil e posteriormente investigado para avaliação de adultos (Santos, 2011). $\mathrm{O}$ teste consiste em ligar letras a números, seguindo a sequência do alfabeto para as letras e respeitando a ordem crescente dos números, por exemplo, 1-A-2-B. Tal teste avalia a habilidade de sequenciamento, atenção visual, habilidades espaciais e motoras simples e flexibilidade cognitiva. Além disso, avalia a atenção sustentada pelo número de ligações corretas realizadas dentro do prazo de um minuto. No presente estudo se 
considerou o desempenho como adequado quando houve 11 ou mais ligações em 60 segundos (Santos, 2011).

Teste de Nomeação de Boston (versão reduzida). Parte integrante da Bateria CERAD foi desenvolvido por Morris et al. (1989) e adaptada para o Brasil por Bertolucci, Okamoto, Toniolo Neto, Ramos e Brucki (1998). É composta por 15 imagens que devem ser nomeadas no prazo de 20 segundos cada. Para cada acerto é atribuído um ponto e se considera que o participante teve um desempenho satisfatório caso tenha nomeado 13 ou mais figuras adequadamente (Bertolucci et al., 1998).

Lista de Palavras. Parte integrante da Bateria CERAD, foi desenvolvido por Morris et al. (1989) e adaptada para o Brasil por Bertolucci et al. (1998). É composto por três tarefas, sendo a primeira a apresentação oral de uma lista de 10 itens para recordação imediata. Tal procedimento é repetido três vezes, com as mesmas palavras sendo apresentadas em diferentes ordens. Como os participantes têm mais de oito anos de estudo, o desempenho é considerado adequando quando recordam 14 palavras ou mais na soma das três apresentações. Após a recordação imediata utiliza-se uma tarefa distratora e após cinco minutos pede-se ao participante que recupere da memória a lista inicialmente apresentada (memória de longo prazo). Nessa etapa a pontuação esperada é de quatro pontos ou mais, sendo atribuído um ponto para cada acerto. A última tarefa é de reconhecimento, nessa etapa se apresenta a listagem inicial misturada com outras 10 palavras e pede-se ao participante que identifique as que estavam na lista original. Espera-se um resultado de oito pontos ou mais para pessoas com maior escolaridade (Bertolucci et al., 1998).

Dígitos. Subteste integrante das Escalas Wechsler de Avaliação de Inteligência Adulta, validado para o Brasil por Nascimento (2000), avalia memória operacional. Consiste na apresentação de uma sequência de números com aumento gradual da quantidade de itens que devem ser recordados inicialmente em ordem direta $\mathrm{e}$ depois em ordem inversa. Considerou-se o desempenho como adequado quando o participante atingiu escore ponderado de 10 , que representa a média prevista para a população brasileira nesse subteste (Nascimento, 2000).

Teste do Desenho do Relógio. Validado para o Brasil por Atalaia-Silva e Lorenço (2008), o teste avalia capacidade de planejamento e organização visoespacial. A atividade consiste em desenhar um relógio de ponteiro marcando um determinado horário e, posteriormente, copiar um relógio desenhado pelo avaliador. Adotouse a correção de 10 pontos, validada para o Brasil por proposta por Atalaia-Silva e Lorenço (2008), na qual a pontuação de zero a cinco mostra déficit e entre seis e 10 pontos é considerada adequada.

Fluência Verbal: Animais. Validado por Dozzibrucki, Malheiros, Okamoto e Bertolucc (1997), consiste em falar o maior número de categorias animais no prazo de um minuto. Os resultados são considerados adequados para pessoas de maior escolaridade quando o respondente relata 13 ou mais categorias de animais.

\section{Treinamento cognitivo}

O treinamento cognitivo consistiu em uma sessão semanal participando de jogos eletrônicos pré-selecionados. As sessões ocorriam uma vez por semana, em dias e horários fixos e pré-determinados entre a equipe de pesquisa e os participantes. As sessões foram iniciadas com 20 minutos (1. a sessão) de duração e houve aumento progressivo de 10 minutos a cada nova sessão, até que atingissem o tempo máximo de 60 minutos (5. ${ }^{\text {a }}$ sessão em diante). Esse tempo (60 minutos) foi determinado para que os jogadores não perdessem o interesse nos jogos por fadiga.

Os jogos disponibilizados para os participantes foram escolhidos previamente pela equipe da pesquisa, com base nos jogos comerciais utilizados em estudos internacionais previamente consultados sobre treinamento cognitivo (referência). As habilidades treinadas por meio desses jogos identificadas nos estudos eram variáveis, mas enfocavam especialmente treinamento de percepção visual, memória e atenção (referência). Foram disponibilizados para os participantes dos dois 
grupos os jogos Need For Speed: The Run, Wii Sports Resort, Wii Party, e New Super Mario Bros.Wii para o console Nintendo Wii, e Guitar Hero 3: Legends of Rock, Prince of Persia The Forgotten Sands e Sports Champions para o console PlayStation 3.

As sessões de treinamento ocorreram em salas previamente agendadas na própria UFTM. A cada sessão de treinamento os participantes podiam escolher livremente entre os jogos disponibilizados, sendo controlados apenas quanto ao tempo de jogo. Os participantes jogavam de forma conjunta, contando com a presença de uma dupla de integrantes da equipe de pesquisa em todas as sessões para controle do tempo e para auxiliar com os videogames, uma vez que a amostra era de não jogadores. As duplas eram formadas por estudantes de Psicologia previamente treinados quanto ao uso dos jogos, dos testes utilizados no presente estudo e sobre funções cognitivas e treino cognitivo. Tais duplas acompanhavam o treino eram fixas por horário e tomou-se o cuidado que a dupla que fizesse as avaliações (pré-teste e pós-teste) não fosse a mesma que acompanhou o treinamento.

Aos dois grupos foi solicitado que não tivessem contato com jogos de videogame durante o tempo de treinamento. Além disso pediu-se que se houvesse contato eventual com jogos de smartphones e/ou tablets em casa, fosse registrado o nome do jogo e o tempo de uso.

\section{Procedimento}

Os estudantes foram convidados por meio de divulgação em sala de aula e adesão voluntária, tomando como cuidado a checagem que os interessados não fossem jogadores de videogame anteriormente. Com aqueles que aceitaram participar do estudo foram agendados horários individuais para realização do pré-teste.

A ordem de aplicação dos testes foi Questionário de Queixa Subjetiva de Memória, Teste de Atenção de Trilhas (Forma B), Lista de Palavras - recordação imediata, Dígitos, Teste do Desenho do Relógio, Lista de Palavras - memória de longo prazo, Lista de Palavras
- reconhecimento, Teste de Nomeação de Boston e Fluência Verbal: Animais. Após a avaliação inicial dos participantes conduziu-se a análise de sua equivalência por meio de teste Mann-Whitney quanto ao desempenho nos testes, sendo identificado que todos poderiam ser classificados como equivalentes. Após essa verificação os participantes foram divididos em dois grupos, sendo o primeiro grupo (G1) o que participaria do treino cognitivo pelo período de cinco a oito semanas e o outro grupo (G2) participaria da intervenção pelo período de nove a 16 semanas. A divisão nos dois grupos teve dupla função, fazer com que cada grupo atue como um grupo controle para o outro e também avaliar o tempo mínimo necessário para que um treinamento cognitivo com jogos de videogame gere algum impacto. Os tempos foram definidos considerando o trabalho de Toril, Reales e Ballesteros (2014), que indica que o tempo de treinamento com jogos eletrônicos sofre grande variação, sendo boa parte deles realizado entre três a quatro meses, mas indicando que resultados distintos foram encontrados nos treinamentos até seis semanas e nos com maior tempo de duração. Assim, decidiu-se manter um grupo com o tempo médio já adotado em outras investigações e manter um grupo com menor número de sessões.

Os dois grupos participaram do treino cognitivo seguindo o protocolo já descrito quanto ao tempo e aos jogos disponibilizados. Após o período de intervenção todos os participantes foram reavaliados, por meio dos mesmos testes utilizados no pré-teste. A reavaliação ocorreu sempre até uma semana após o período de treinamento se encerrar.

O estudo foi aprovado no Comitê de Ética em Pesquisa da Universidade Federal do Triângulo Mineiro (CEP 1633/2010) e todos os participantes assinaram o Termo de Consentimento Livre e Esclarecido.

\section{Análise de dados}

Foram feitas medidas descritivas de porcentagem, tendência central e dispersão para caracterização da amostra e dos desempenhos dos participantes na ava- 
liação pré e pós-intervenção. Para avaliar a efetividade da intervenção realizou-se a comparação entre os desempenhos dos jogadores no pré e pós-teste, por meio do teste de Wilcoxon para amostras pareadas. Além disso, para verificar a influência do tempo de jogo foi feita comparando o G1 e o G2 no pós-teste, por meio do teste de Mann-Whitney para amostras independentes. Todas as análises consideraram significância de 5\%.

\section{Resultados}

$\mathrm{Na}$ avaliação pré-treinamento se observou que parte dos participantes não tinha queixa de memória $(\mathrm{n}=06)$ ou tinha queixa leve $(\mathrm{n}=08)$, totalizando $56 \%$. $\mathrm{Na}$ avaliação objetiva de memória, os participantes recordaram imediatamente 21,56 palavras (DP. 2,98) e 7,52 palavras (DP. 1,42) na tarefa de recordação tardia, ficando todos com classificação normal para memória imediata e de longo prazo (Tabela 1). Recordaram 9,28 palavras em média para a tarefa de reconhecimento (DP. 1,48), ficando 88\% deles com classificação normal.

A memória de trabalho foi avaliada por meio do número de dígitos recordados e os resultados mostraram média de 6,24 números na ordem direta (DP. 0,93) e 4,48 (DP. 1,89) na ordem inversa. Esses resultados mostraram estar dentro do esperado para $96 \%$ dos participantes na ordem direta e $88 \%$ na ordem inversa. Outro aspecto observado é que $92 \%$ dos participantes mostraram efeito de aprendizado, recordando mais palavras na tarefa de recordação imediata quando a lista de palavras era reapresentada por até três vezes. Além disso, observou-se que houve média de 0,40 intrusões de palavras (DP. 0,50) na tarefa de recordação imediata e de 0,24 (DP. 0,43$)$ na recordação tardia.

Ao avaliar a atenção observou-se uma média de 14,44 ligações (DP. 6,89), ficando 19 participantes com classificação normal $(76,0 \%)$. Para a capacidade de planejamento e percepção visual, observou-se pontuação média de 7,88 para a tarefa de evocação (DP. 2,28) e 9,36 (DP. 1,47) para a tarefa de cópia. Esse resultado representa $72 \%$ de respostas normais para a tarefa de evocação. Quando houve pista externa (na tarefa de cópia), as respostas foram consideradas normais em $96 \%$ dos casos.

A linguagem foi avaliada por meio dos testes de fluência verbal e nomeação. Com relação à fluência verbal, os participantes falaram em média 21,20 palavras em 60 segundos (DP. 4,74), sendo que 92\% receberam classificação normal. Conseguiram nomear corretamente 14,40 imagens em média (DP. 0,64), ficando todos classificados como normais para essa habilidade.

Para o G1 o tempo de treinamento ficou em 380 minutos, enquanto para o G2 esse tempo foi de 860 minutos de jogos supervisionados. Ao longo dos encontros da intervenção os jogadores escolheram jogar New Super Mario Bros e Guitar Hero 3: Legends of Rock em todas as sessões de treinamento, os demais jogos foram usados apenas em alguns encontros. Após esse período foi realizada a reavaliação dos participantes e seus resultados estão disponibilizados na Tabela 1. Nessa reavaliação, ao considerar todos os participantes, observou-se ausência de queixa ou queixa leve de memória em 13 participantes (52\%), valor próximo ao do pré-teste, não mostrando diferenças significativas quanto ao pré e pós-teste. Mas o número de pessoas com queixa acentuada diminuiu de quatro para duas pessoas.

Todos os participantes mantiveram a capacidade de recordação imediata normal, mas houve incremento significativo $(\mathrm{W}=-3,55 ; \mathrm{p}<0,001)$ no número de palavras recordadas, ficando a média da reavaliação em 25,32 (DP. 2,66). Houve uma melhoria na capacidade de recordação tardia também $(\mathrm{W}=-3,75 ; \mathrm{p}<0,001)$, que foi avaliada com média de 9,04 (DP. 0,93) no pós-teste. A capacidade de reconhecimento também recebeu um incremento, sendo avaliada em 9,88 em média (DP. 0,33 ), o que mostra uma melhoria significativa com relação ao pré-teste $(\mathrm{W}=-2,20 ; \mathrm{p}=0,028)$.

Quanto à memória operacional, a média de dígitos recordados em ordem direta ficou em 6,56 (DP. 0,82) e 4,72 (DP. 0,89) na ordem indireta. Esses resultados mostraram melhora no desempenho dos participantes 
na recordação de dígitos em ordem inversa $(\mathrm{W}=-3,97$; $\mathrm{p}<0,001)$, mas não na ordem direta $(\mathrm{W}=-1,60 ; \mathrm{p}=$ 0,109). Observou-se efeito de aprendizado para $88 \%$ dos participantes na reavaliação e não houve diferença entre essa capacidade no pré e pós-teste. Houve uma média 0,36 intrusões na reavaliação na tarefa de recordação imediata (DP. 0,49) e 0,20 para recordação tardia (DP. 0,41), o que não indica diferença entre pré e pós-teste.

Ao avaliar a atenção, os participantes fizeram em média 18,16 ligações no pós-teste (DP. 6,40), ficando $88 \%$ dos participantes com classificação normal, o que mostrou melhoria no desempenho após a intervenção
$(\mathrm{W}=-2,45 ; \mathrm{p}=0,014)$. Ao analisar a capacidade de planejamento e percepção visual, resultados adequados foram observados em $76 \%$ dos participantes na tarefa de evocação ( $=7,56$; DP 2,45$)$ e $96 \%$ na cópia $(=9,12$; DP 1,39), não mostrando melhoria após o treinamento.

A capacidade para nomear corretamente as imagens mostradas teve um incremento na média $(=14,84$; DP $=0,37)$ e manteve a classificação de normalidade para todos os participantes, o que indicou uma melhora significativa $(\mathrm{W}=-2,84 ; \mathrm{p}=0,005)$. A média de palavras ditas ficou em 22,72 palavras (DP. 4,45), mas esse incremento não foi considerado significativo estatisticamente.

Tabela 1. Dados descritivos e diferenças entre avaliações. $(n=25)$.

\begin{tabular}{|c|c|c|c|c|}
\hline Variável & $\begin{array}{c}\text { Avaliação } \\
\text { n (\%) / } \bar{x}[\text { D.P }]\end{array}$ & $\begin{array}{c}\text { Reavaliação } \\
\text { n (\%) / } \bar{x}[\text { D.P] }\end{array}$ & Teste Wilcoxon & p-valor \\
\hline \multicolumn{5}{|l|}{ Queixa de Memória } \\
\hline Média & $4,96[3,12]$ & $5,32[3,29]$ & $-0,92$ & 0,357 \\
\hline Sem Queixa & $06(24,00)$ & $05(20,00)$ & & \\
\hline Leve & $08(32,00)$ & $08(32,00)$ & & \\
\hline Moderada & $07(28,00)$ & $10(40,00)$ & & \\
\hline Acentuada & $04(16,00)$ & $02(8,00)$ & & \\
\hline Recordação Imediata & & & $-3,55$ & $<0,001$ \\
\hline Média & $21,56[2,99]$ & $25,32[2,66]$ & & \\
\hline Normal & $25(100,00)$ & $25(100,00)$ & & \\
\hline Deficit & $00(00,00)$ & $00(00,00)$ & & \\
\hline \multicolumn{5}{|l|}{ Recordação Tardia } \\
\hline Média & $7,52[1,42]$ & $9,04[0,93]$ & $-3,75$ & $<0,001$ \\
\hline Normal & $25(100,00)$ & $25(100,00)$ & & \\
\hline Déficit & oo $(00,00)$ & $00(00,00)$ & & \\
\hline \multicolumn{5}{|l|}{ Reconhecimento } \\
\hline Média & $9,28[1,49]$ & $9,88[0,33]$ & $-2,20$ & 0,028 \\
\hline Normal & $22(88,00)$ & $25(100,00)$ & & \\
\hline Déficit & $03(12,00)$ & oo $(00,00)$ & & \\
\hline \multicolumn{5}{|l|}{ Dígitos } \\
\hline Média - Ordem Direta & $6,24[0,92]$ & $6,56[0,82]$ & $-1,60$ & 0,109 \\
\hline Normal & $24(96,00)$ & $25(100,00)$ & & \\
\hline Déficit & $01(4,00)$ & oo $(00,00)$ & & \\
\hline Média - Ordem Indireta & $4,48[1,04]$ & $4,72[0,89]$ & $-3,97$ & $<0,001$ \\
\hline
\end{tabular}




\begin{tabular}{|c|c|c|c|c|}
\hline Variável & $\begin{array}{c}\text { Avaliação } \\
\text { n (\%) / } \bar{x}[D . P]\end{array}$ & $\begin{array}{c}\text { Reavaliação } \\
\text { n (\%) / X [D.P] }\end{array}$ & Teste Wilcoxon & p-valor \\
\hline Normal & $22(88,00)$ & $25(100,00)$ & & \\
\hline Déficit & $03(12,00)$ & $00(00,00)$ & & \\
\hline Aprendizagem & & & $-0,58$ & 0,564 \\
\hline Sim & $23(92,00)$ & $22(88,00)$ & & \\
\hline Não & $02(8,00)$ & $03(12,00)$ & & \\
\hline Trilhas & & & $-2,45$ & 0,014 \\
\hline Média & $14,44[6,89]$ & $18,16[6,40]$ & & \\
\hline Normal & $19(76,00)$ & $22(88,00)$ & & \\
\hline Déficit & $06(24,00)$ & $03(12,00)$ & & \\
\hline \multicolumn{5}{|l|}{ Relógio } \\
\hline Média - Evocação & $7,88[2,28]$ & $7,56[2,45]$ & $-0,55$ & 0,582 \\
\hline Normal & $18(72,00)$ & $19(76,00)$ & & \\
\hline Déficit & $07(28,00)$ & $06(24,00)$ & & \\
\hline Média - Cópia & $9,36[1,47]$ & $9,12[1,39]$ & $-1,46$ & 0,144 \\
\hline Normal & $24(96,00)$ & $24(96,00)$ & & \\
\hline Déficit & $01(4,00)$ & $01(4,00)$ & & \\
\hline \multicolumn{5}{|l|}{ Intrusões } \\
\hline Recordação imediada & $0,40[0,50]$ & $0,36[0,49]$ & $-0,28$ & 0,782 \\
\hline Recordação tardia & $0,24[0,43]$ & $0,20[0,41]$ & & \\
\hline Nomeação & & & $-2,84$ & 0,005 \\
\hline Média & $14,40[0,64]$ & $14,84[0,37]$ & & \\
\hline Normal & $25(100,00)$ & $25(100,00)$ & & \\
\hline Déficit & oo $(00,00)$ & $00(00,00)$ & & \\
\hline \multicolumn{5}{|l|}{ Fluência Verbal } \\
\hline Média & $21,20[4,74]$ & $22,72[4,45]$ & $-1,51$ & 0,130 \\
\hline $\begin{array}{l}\text { Normal } \\
\text { Déficit }\end{array}$ & $\begin{array}{c}23(92,00) \\
02(8,00)\end{array}$ & $\begin{array}{c}25(100,00) \\
00(00,00)\end{array}$ & & \\
\hline
\end{tabular}

Os resultados intragrupo separadamente por grupo encontram-se apresentados nas Tabelas 2 (G1) e Tabela 3 (G2). Observou-se que os integrantes do G1 melhoraram no pós-teste quanto a habilidade de recordação imediata $(\mathrm{W}=-2,63 ; \mathrm{p}=0,008)$, recordação tardia $(\mathrm{W}=$ $-2,62 ; p=0,009)$ e atenção (W= $-2,65 ; p=0,008)$. No grupo G2 observou-se melhoria na recordação imediata $(\mathrm{W}=-2,35 ; \mathrm{p}=0,019)$, recordação tardia $(\mathrm{W}=-2,67$; $\mathrm{p}=0,008)$, capacidade de memorização de dígitos em ordem inversa $(\mathrm{W}=-1,94 ; \mathrm{p}=0,052)$ e capacidade de nomeação $(\mathrm{W}=-2,30 ; \mathrm{p}=0,021)$. 
Tabela 2. Comparação intragrupo pré e pós-intervenção para treinamento de 8 semanas $(\mathrm{n}=11)$

\begin{tabular}{|l|c|c|c|c|}
\hline \multicolumn{1}{|c|}{ Variável } & Pré-teste & G1 & W & p-valor \\
\hline Queixa de Memória & $4,36(3,04)$ & $4,64(2,62)$ & $-0,77$ & 0,457 \\
\hline Recordação Imediata & $20,73(3,49)$ & $25,45(1,75)$ & $-\mathbf{2 , 6 3}$ & $\mathbf{0 , 0 0 8}$ \\
\hline Recordação Tardia & $7,36(1,21)$ & $9,09(0,83)$ & $-\mathbf{2 , 6 2}$ & $\mathbf{0 , 0 0 9}$ \\
\hline Reconhecimento & $9,55(1,04)$ & $10,00(0,00)$ & $-\mathbf{1 , 3 4}$ & 0,180 \\
\hline Dígitos - Ordem Direta & $6,36(1,12)$ & $6,45(0,93)$ & $-0,28$ & 0,783 \\
\hline Dígitos - Ordem Inversa & $4,55(1,13)$ & $4,82(0,87)$ & $-0,79$ & 0,429 \\
\hline Trilhas & $16,09(4,96)$ & $21,64(1,80)$ & $-\mathbf{2 , 6 5}$ & $\mathbf{0 , 0 0 8}$ \\
\hline Relógio - Evocação & $8,45(2,25)$ & $7,36(2,38)$ & $-1,37$ & 0,170 \\
\hline Relógio - Cópia & $9,73(0,65)$ & $9,00(1,79)$ & $-1,51$ & 0,131 \\
\hline Intrusões - Recordação imediata & $0,36(0,50)$ & $0,27(0,47)$ & $-0,45$ & 0,655 \\
\hline Intrusão - Recordação Tardia & $0,27(0,47)$ & $0,27(0,47)$ & 0,00 & $\mathbf{1 , 0 0 0}$ \\
\hline Fluência Verbal & $20,91(4,61)$ & $22,73(31,01)$ & $-0,89$ & 0,372 \\
\hline Nomeação & $14,64(0,50)$ & $\mathbf{1 4 , 9 1 ( 0 , 3 0 )}$ & $-1,73$ & 0,083 \\
\hline
\end{tabular}

${ }^{1}=$ Média e Desvio-Padrão; ${ }^{2}=$ Teste Wilcoxon para amostras pareadas

Tabela 3. Comparação intragrupo pré e pós-intervenção para treinamento de 9 a 16 semanas $(n=14)$

\begin{tabular}{|c|c|c|c|c|}
\hline Variável & Pré-teste & $\mathbf{G 2}^{1}$ & $\mathbf{W}^{2}$ & p-valor \\
\hline Queixa de Memória & $5,43(3,20)$ & $5,86(3,73)$ & $-0,92$ & 0,359 \\
\hline Recordação Imediata & $22,21(2,45)$ & $25,21(3,26)$ & $-2,35$ & 0,019 \\
\hline Recordação Tardia & $7,64(1,60)$ & $9,00(1,04)$ & $-2,67$ & 0,008 \\
\hline Reconhecimento & $9,07(1,77)$ & $9,79(0,43)$ & $-1,73$ & 0,084 \\
\hline Dígitos - Ordem Direta & $6,14(0,77)$ & $6,64(0,93)$ & $-0,36$ & 0,716 \\
\hline Dígitos - Ordem Inversa & $4,43(1,01)$ & $9,21(1,05)$ & $-1,94$ & 0,052 \\
\hline Trilhas & $13,14(8,04)$ & $15,43(7,41)$ & $-1,08$ & 0,278 \\
\hline Relógio - Evocação & $7,43(2,28)$ & $7,71(2,58)$ & $-0,25$ & 0,798 \\
\hline Relógio - Cópia & $9,07(1,86)$ & $9,21(1,05)$ & $-0,70$ & 0,483 \\
\hline Intrusões - Recordação imediata & $0,43(0,51)$ & $0,43(0,51)$ & 0,00 & 1,000 \\
\hline Intrusão - Recordação Tardia & $0,21(0,43)$ & $0,14(0,36)$ & $-0,45$ & 0,655 \\
\hline Fluência Verbal & $21,43(5,00)$ & $22,71(5,40)$ & $-1,18$ & 0,235 \\
\hline Nomeação & $14,21(0,70)$ & $14,79(0,43)$ & $-2,30$ & 0,021 \\
\hline
\end{tabular}

${ }^{1}=$ Média e Desvio-Padrão; ${ }^{2}=$ Teste Wilcoxon para amostras pareadas 
Além disso, ao comparar o desempenho dos dois grupos no pós-teste, observou-se que apenas os grupos diferiram apenas quanto a habilidade de atenção $(\mathrm{U}=$
31,00; $p=0,010)$. Nessa medida o G1 mostrou melhor desempenho em relação ao grupo G2.

Tabela 4. Comparação dos grupos após a intervenção por tempo de intervenção. $(n=25)$

\begin{tabular}{|c|c|c|c|c|c|}
\hline Variável & Pré-teste & $\mathbf{G 1}^{1}$ & $\mathbf{G 2}^{1}$ & $\mathbf{U}^{2}$ & p-valor \\
\hline Queixa de Memória & $5,32(3,29)$ & $4,64(2,62)$ & $5,86(3,73)$ & 64,00 & 0,501 \\
\hline Recordação Imediata & $25,32(2,66)$ & $25,45(1,75)$ & $25,21(3,26)$ & 71,00 & 0,738 \\
\hline Recordação Tardia & $9,04(0,93)$ & $9,09(0,83)$ & $9,00(1,04)$ & 76,50 & 0,977 \\
\hline Reconhecimento & $9,88(0,33)$ & $10,00(0,00)$ & $9,79(0,43)$ & 60,50 & 0,109 \\
\hline Dígitos - Ordem Direta & $6,56(0,82)$ & $6,45(0,93)$ & $6,64(0,93)$ & 68,50 & 0,611 \\
\hline Dígitos - Ordem Inversa & $4,72(0,89)$ & $4,82(0,87)$ & $9,21(1,05)$ & 66,50 & 0,529 \\
\hline Trilhas & $18,16(6,40)$ & $21,64(1,80)$ & $15,43(7,41)$ & 31,00 & 0,010 \\
\hline Relógio - Evocação & $7,56(2,45)$ & $7,36(2,38)$ & $7,71(2,58)$ & 66,50 & 0,545 \\
\hline Relógio - Cópia & $9,12(1,39)$ & $9,00(1,79)$ & $9,21(1,05)$ & 75,50 & 0,928 \\
\hline Intrusões - Recordação imediata & $0,36(0,49)$ & $0,27(0,47)$ & $0,43(0,51)$ & 65,00 & 0,430 \\
\hline Intrusão - Recordação Tardia & $0,20(0,41)$ & $0,27(0,47)$ & $0,14(0,36)$ & 67,00 & 0,430 \\
\hline Fluência Verbal & $22,72(4,45)$ & $22,73(31,01)$ & $22,71(5,40)$ & 73,50 & 0,847 \\
\hline Nomeação & $14,84(0,37)$ & $14,91(0,30)$ & $14,79(0,43)$ & 67,50 & 0,413 \\
\hline
\end{tabular}

${ }^{1}=$ Média e Desvio-Padrão; ${ }^{2}=$ Teste Mann Whitney

\section{Discussão}

Este trabalho visou avaliar a efetividade de um treinamento cognitivo utilizando jogos eletrônicos como forma de melhorar as habilidades de atenção e memória de estudantes universitários, mostrando o impacto positivo do treinamento nessas habilidades e na capacidade de nomeação. Esses resultados apontam no mesmo sentido de estudos anteriores sobre uso de jogos em treinamentos cognitivos (Steenbergen, Sellaro, Stock, Beste, \& Colzato, 2015; Kristjánsson, 2013; Chiappe, Conger, Liao, Caldwell, \& Vu, 2013; Mishra, Bavelier, \& Gazzaley, 2012; Alves \& Carvalho, 2010; Ray et al., 2017).
A tarefa de atenção solicitada no presente trabalho envolvia localizar e ligar letras e números seguindo uma ordem e o sucesso nessa tarefa implica ignorar os demais estímulos apresentados. Os participantes do treinamento melhoraram o seu desempenho nessa tarefa, o que corrobora com achados sobre o perfil atencional de jogadores de videogame. Segundo Bavelier et al. (2012), quando jogadores de videogame são comparados cognitivamente com não jogadores, os primeiros mostram desempenho superiores na atenção, conseguindo localizar alvos com mais agilidade e menor interferência de estímulos distratores pelo tipo de habilidade demandada pelos jogos. As característica dos jogos, 
com a presença de vários elementos de cor, luz, som e textura auxiliam no treinamento dessas funções (Toril, Reales, \& Ballesteros, 2014), em especial a habilidade de rastreio visual e foco atencional nos jogadores.

Com o treinamento cognitivo os participantes tiveram melhoria na memória imediata, memória tardia e reconhecimento. Ray et al. (2017) indica que os jogadores de jogos eletrônicos apresentam uma melhora em atenção visual, resolução de problemas, flexibilidade cognitiva, memória episódica, memória de trabalho e orientação espacial quando comparados a não jogadores.

Quando os grupos foram comparados separadamente, independentemente do tempo de intervenção, foi possível notar melhora no desempenho de memória imediata e memória tardia, mas só houve melhora na memória de trabalho dos participantes que treinaram entre 9 e 16 semanas. Embora seja condizente com os resultados de estudos anteriores sobre memória, uma possível explicação para observarmos melhora na memória de trabalho apenas do grupo que treinou por mais tempo se deva a escolha por fazer apenas uma sessão semanal de treinamento.

Os trabalhos de Nouchi et al. (2013) e Boot et al. (2008) apontaram melhoria na memória de trabalho e memória de curto prazo com grupos com diferentes tempos de treinamento, mas apenas com participantes que treinavam mais vezes por semana. No estudo de Boot et al. (2008), o treinamento foi feito em 15 sessões, distribuídas ao longo de cinco semanas, enquanto no estudo de Nouchi et al. (2013) o treinamento foi feito durante 20 sessões divididas em quatro semanas.

Memória, atenção, habilidade para multitarefas e percepção visual têm sido as funções que demonstram mais impacto positivo com treinamentos com jogos eletrônicos (Mishra et al., 2012), embora muitos estudos deixem dúvidas sobre diferenças no nível das habilidades prévias dos participantes (Kristjánsson, 2013). Gnambs e Appel (2017) apontam que uma limitação dos estudos sobre treinamento cognitivo com jogos parte do fato que os grupos de jogadores poderiam não ser equivalentes previamente e que os jogadores são tratados de forma indiscriminada, sem considerar o tempo dedicado ao jogo semanalmente. Esse foi um dos aspectos que se buscou controlar no presente estudo, investigando previamente a equivalência entre os participantes e optou-se por investigar apenas pessoas que não tinham prática prévia com jogos de videogame. Assim, a melhoria no desempenho do teste de nomeação, memória e atenção permitem perceber que o treinamento com jogos eletrônicos foi eficaz.

No presente estudo, evidenciou-se ainda melhora no desempenho de nomeação. Poucos estudos investigaram como os videogames impactam habilidades de linguagem, sendo difícil identificar se esse foi um achado isolado ou se essa habilidade pode ser efetivamente melhorada com a prática de jogos eletrônicos. Contudo, Gnambs e Appel (2017) observaram ganhos na capacidade de compreensão de palavras ouvidas em jogadores, independentemente do tempo semanal dedicado a jogar.

Não foram observadas melhorias nas queixas de memória dos participantes após a intervenção. Os participantes já haviam indicado que tinham uma percepção mediana sobre a sua memória desde o pré-teste e a não alteração após o treinamento pode refletir uma característica da amostra, que era composta por jovens universitários, pessoas funcionais e sem diagnósticos, assim, pouco sujeitas a influências externas quanto a essa percepção. Além disso, também não foram observados implementos no desempenho para a recordação de dígitos em ordem direta, capacidade de planejamento, presença de intrusões e capacidade de fluência verbal. Esses achados indicam que o treino cognitivo pode não ser efetivo para treinar todas as habilidades cognitivas ou que adaptações podem ser requeridas quanto aos jogos, frequência e tempo de treinamento caso se deseje treinar diferentes funções. Outros estudos também observaram habilidades que não foram melhoradas por meio de treinamento cognitivo, Wentink et al. (2016) não perceberam melhoria na capacidade de planejamento e Gnambs e Appel (2017) não observaram impacto do treinamento 
sobre as habilidades de linguagem de participantes que passaram por intervenções com jogos eletrônicos.

\section{Conclusões}

Considerando os resultados observados, houve melhoria nas habilidades de memória, atenção e nomeação com o treinamento com jogos de videogame. Esses resultados são animadores e apontam para a relevância de mais estudos na área para desenvolvimento de protocolos padronizados de treinamento para diferentes habilidades e populações. Contudo, precisam ser relativizados pois esse estudo apresenta limitações que podem ter interferido com os resultados observados. Embora contribua com informações brasileiras para uma temática ainda pouco investigada no País, a amostra da pesquisa foi pequena e restrita a estudantes de uma única instituição. Além disso, o tempo de realização da intervenção não foi padronizado para todos os participantes, ficando entre cinco e oito semanas para um grupo e entre nove e 16 para o outro. Com isso a influência do tempo de jogo não pôde ser mais bem esclarecida. Sugere-se para pesquisas futuras que o tempo de treinamento seja reduzido em número de semanas e que haja mais sessões de treinamento por semana, visando a superação dessa limitação. Outra limitação referiu-se a medida de autorrelato para acompanhamento de uso de jogos fora dos momentos de treinamento. Não foram conduzidas análises que considerassem os jogos que os participantes utilizavam fora do horário de treinamento, o que representa um possível viés que não foi controlado ao longo da investigação. Uma solução para essa limitação poderia ser o uso de um checklist básico de perguntas a serem feitas semanalmente aos participantes sobre os seus hábitos ao longo da semana.

\section{Referências}

Alves, L., \& Carvalho, A. M. (2010). Videogame e sua influência em teste de atenção. Psicologia em Estudo, 15(3), 519-525. https://doi.org/10.1590/s1413$\underline{73722010000300009}$

Aranha, G. (2006). Jogos eletrônicos como um conceito chave para o desenvolvimento de aplicações imersivas e interativas para o aprendizado. Ciências \& Cognição, 7, 105-110.

Atalaia-Silva, K.C., \& Lourenço, R. A. (2008). Tradução, adaptação e validação de construto do Teste do Relógio aplicado entre idosos no Brasil. Revista de Saúde Pública, 42(5), 930-937. https://doi.org/10.1590/s0034$\underline{89102008000500020}$

Baum, C., \& Maraschin, C. (2017). Level up! Desenvolvimento cognitivo, aprendizagem enativa e videogames. Psicologia \& Sociedade, 29, 1-11. https:// doi.org/10.1590/1807-0310/2017v29132334

Ballestros, S., Mayas, J., Ruiz-Marques, E., Pietro, A., ... Reales Avilés, J. M. (2017). Effects video game training on behavioral and electrophysiological measures of attention and memory: protocol for a randomized controlled trial. JMRI Publications, 6(1), 1-20. https:// doi.org/10.2196/resprot.6570

Bavelier, D., Achtman, R., \& Föcker, M. (2012). Neural bases of selective attention in action video game players. Vision Research 61, 132-143. https://doi.org/10.1016/j. visres.2011.08.007

Bertolucci, P. H. F., Okamoto, I. H., Toniolo Neto, J., Ramos, L. R., \& Brucki, S. M. D. (1998). Desempenho da população brasileira na bateria neuropsicológica do Consortium to Establish a Eegistry for Alzheimer's Disease (CERAD). Revista de Psiquiatria Clínica, 25(2), 80-83.

Boot W. R., Basak C., Erickson K. I., Neider M., Simons D. J., Fabiani M., ... Kramer A. F. (2010). Transfer of skill engendered by complex task training under conditions of variable priority. Acta Psycholica, 135, 349-357. https://doi.org/10.1016/j.actpsy.2010.09.005

Boot, W. R., Kramer, A. F., Simons, D. J., Fabiani, M., \& Gratton, G. (2008). The effects of video game playing on attention, memory, and executive control. Acta Psychologica, 129, 387-398. https://doi.org/10.1016/j. actpsy.2008.09.005 
Colzato, L. S., van den Wildenberg, W. P. M., Zmigrod, S., \& Hommel, B. (2012). Action video gaming and cognitive control: playing first person shooter games is associated with improvement in working memory but not action inhibition. Psychological Research, 77(2), 234-239. https://doi.org/10.1007/s00426-012-0415-2

Cutter, C. J., Schottenfeld, R. S., Moore, B. A., ... Barry D. T. (2014). A pilot trial of a videogame-based exercise program for methadone maintained patients. Journal of Substance Abuse Treatment, 47(4), 299-305. https:// doi.org/10.1016/j.jsat.2014.05.007

Chiappe, D., Conger, M., Liao, J., Caldwell, J. L., \& Vu, k. P. L. (2013). Improving multi-tasking ability through action videogames. Applied Ergonomics, 44, 278-284. https://doi.org/10.1016/j.apergo.2013.01.005

Chisholm, J. D., Hickey, C., Theeuwes, J., \& Kingstone, A. (2010). Reduced attentional capture in action video game players. Attention, Perception, \& Psychophysics, 72(3), 667-671. https://doi.org/10.3758/app.72.3.667

Conners' K. C., \& MHS Staff (2006). Conner's Continuous Performance Test (CPT II) - Technical Guide and Software Manual. Canada: MHS. DOI inexistente

Draganski B., Gaser C., Kempermann G, Kuhn, H.G., Winkler, J., Büchel, C., \& May, A. (2006). Temporal and spatial dynamics of brain structure changes during extensive learning. Journal of Neuroscience, 26(63), 14-7. https://doi.org/10.1523/jneurosci.4628-05.2006

Dozzibrucki, S. M., S. M. F., Malheiros, I. H. Okamoto, \& Bertolucc, P. H. F. (1997). Dados normativos para o Teste de Fluência Verbal categorias animais em nosso meio. Arquivo de Neuropsiquiatria, 55(1), 56 - 61. https://doi.org/10.1590/s0004-282x1997000100009

Dye, M.W. G., \& Bavelier, D. (2010). Differential development of visual attention skills in school-age children. Vision Research, 50, 452-459. https://doi.org/10.1016/j. visres.2009.10.010

Francis, D. B., Cornello, M. L. G., \& Marshall, L. H. (2016). How does gameplaying support values and psychological well-being among cancer survivors? Games for health journal: research, development, and clinical applications, 5(2), 1-7. https://doi.org/10.1089/ g4h.2015.0044
Gnambs, T., \& Appel, M. (2016). Is computer gaming associated with cognitive abilities? A population study among German adolescents. Intelligence, 1-10. https:// doi.org/10.1016/j.intell.2016.12.004

Green, C. S., \& Bavelier, D. (2006). Action video game experience alters the spatial resolution of attention. Psychological Science, 18, 88 - 94. https://doi. org/10.1111/j.1467-9280.2007.01853.x

Klingberg, T. (2010). Training and plasticity of working memory. Trends in Cognitive Sciences, 14, 317-324. https://doi.org/10.1016/j.tics.2010.05.002

Kristjánsson, A. (2013). The case for causal influences of action videogame play upon vision and attention. Attention, Perception \& Psychophysics, 75, 667-672. https://doi.org/10.3758/s13414-013-0427-z

Leiker, A. M., Bruzi, A. T., Miller, M. W., Nelson, M., Wegman, R., \& Lohse, K. R. (2016). The effects of autonomous difficulty selection on engagement, motivation, and learning in a motion-controlled video game task. Human Moviment Science, 49, 326-335. https://doi.org/10.1016/j.humov.2016.08.005

Li, R., Polat, U., Scalzo, F., \& Bavelier, D. (2010). Reducing backward masking through action game training. Journal of Vision, 10(14), 1-13. https://doi. org/10.1167/10.14.33

Miotto, E. C., Sato, J., Lúcia, M. C. S., Camargo, C. H. P., \& Scaff, M. (2010). Desenvolvimento de uma versão adaptada do Boston Naming Test para a língua portuguesa. Revista Brasileira de Psiquiatria, 32 (3), 279 -282. https://doi.org/10.1590/s1516-44462010005000006

Morris, J. C., Heyman, A., Mohs, R. C., Hughs, J. P., Van Belle, G., Fillenbaum, G., ... Clark, C. (1989). The Consortium to Establish a Registry for Alzheimer's Disease (CERAD): Part I. Clinical and neuropsychological assessment of Alzheirmer's disease. Neurology, 9, 1159-1165.https://doi.org/10.1212/wn1.39.9.1159

Montiel, J. M., \& Capovilla, A. G. S. (2009). Teste de Trilhas. Em A. G.S. Capovilla \& F.C. Capovilla (Orgs.), Teoria e pesquisa em avaliação neuropsicológica (pp. 94-95). São Paulo: Memnon. 
Misha, J., Bavelier, D., \& Gazzaley, A. (2012). How to assess gaming - induced benefits on attention and working memory. Games for health journal: research, development, and clinical applications, 1(3), 192-198. https://doi.org/10.1089/g4h.2011.0033

Nouchi, R., Taki, Y., Takeuchi, H., Hashizume, H., Nozawa, T., ... Kawashima R. (2013). Brain Training Game Boosts Executive Functions, Working Memory and Processing Speed in the Young Adults: A Randomized Controlled Trial. PLoS ONE, 8(2), 1-13. https://doi.org/10.1371/journal.pone.0055518

Neubauer, A. C., Bergner, S., \& Schatz, M. (2010). Two vs. three dimensional presentation of mental rotation tasks: Sex differences and effects of training on performance and brain activation. Intelligence, 38, 529-539. https://doi.org/10.1016/j.intell.2010.06.001

Oliveira, V. B. (2007). Jogos de regras e a resolução de problemas. Petrópolis:Vozes.

Ray, N. R., O’Connell, M. A., Nashiro, K., Smith, R. T., Qin, S., \& Basak, C. (2017). Evaluating the relationship between white matter integrity, cognition, and varieties of video game learning. Restorative Neurology and Neuroscience, 35, 437-456. https://doi.org/10.3233/rnn-160716

Redick, T. S., \& Webster, S. B. (2014). Videogame interventions and spatial ability interactions. Frontiers in Human Neuroscience, 8(183), 1-5. https://doi. org/10.3389/fnhum.2014.00183

Santos, J. (2011). Validação do Teste de Trilhas B para uso em pacientes brasileiros com câncer em cuidados paliativos. Dissertação de mestrado, Universidade de São Paulo. https://doi.org/10.11606/d.7.2011.tde$\underline{18082011-074222}$

Severini, G., Straudi, S., Pavarelli, C., Roit, M. D., \& Basaglia N. (2017). Use of Nintendo Wii Balance Board for posturografic analysis of multiple sclerosis patients with minimal balance impairment. Journal of NeuroEngineering and Rehabilitation, 14(19), 1-14. https://doi.org/10.1186/s12984-017-0230-5

Suzuki, F. T. I., Matias, M. V., Silva, M. T. A., \& Oliveira, M. P. M. T. (2009). O uso de videogames, jogos de computador, e internet por uma amostra de universitários da Universidade de São Paulo. Jornal Brasileiro de Psiquiatria, 58(3), 162-168. https://doi. org/10.1590/s0047-20852009000300004
Scholz, J., Klein, M. C., Behrens, T. E. J., \& JohansenBerg, H. (2009). Training induces changes inwhite-matter architecture. Nature Neuroscience, 12(11), 1370-1371. https://doi.org/10.1038/nn.2412

Steenbergen, L., Sellaro, R., Stock, A. K., Beste, C., \& Colzato, L. S. (2015). Action video gaming and cognitive control: playing first person shooter game is associated with improved cascading but not inhibition. PLoS ONE, 10(12), 1-15. https://doi.org/10.1371/journal.pone.0144364

Tobiansky, R., Blizard, R., Livingston, G., \& Mann. (1995). The Gospeal Oak Study stage IV: the clinical relevance of subjective memory impairment in older people. Psychological Medicine, 25(4), 779-786. https:// doi.org/10.1017/s0033291700035029

Thompson, D., Bhatt, R., Vazquez, I., Cullen, K. W., ... Liu, Y. (2015). Creating action plans in a serious video game increases and maintains child fruit-vegetable intake: a randomized controlled trial. International Journal of Behavioral Nutrition and Physical Activity, 12(39), 2-10. https://doi.org/10.1186/s12966-015-0199-Z

Thorell, L. B., Lindqvist S., Bergman Nutley S., Bohlin G., Klingberg T. (2009). Training and transfer effects of executive functions in preschool children. Dev. Sci. 12, 106-113. https://doi.org/10.1111/j. 1467-7687.2008.00745.x

Toril, P., Reales, J. M., \& Ballesteros, S. (2014). Video game training enhances cognition of older adults: A meta-analytic study. Psychology and Aging, 29(3), 706-716. https://doi.org/10.1037/a0037507

Wentik, M. M., Berger, M. A. M., Kloet, A. J., ... Vliet Vlieland, T. P. M. (2016). The effects of an 8-week computer-based brain training programme on cognitive functioning, QoL, and sef-efficacy after stroke. Neuropsichological Rehabilitation, 4(45), 1-19. https:// doi.org/10.1080/09602011.2016.1162175

\section{Recebido em: 15/12/2017.}

Aprovado em: 25/6/2019.

Publicado em: $\mathrm{xx} / \mathrm{x} / \mathrm{xxxx}$

Endereço para correspondência: Sabrina Martins

Barroso (Rua Carmelita Rezende, №70, Uberaba, Uberaba-MG, CEP: 38.081. 480; (34)3317-4260/

(34)9991-0850; smb.uftm@gmail.com). 


\section{Nome: Fernanda Esteves Pereira}

E-mail: fernandaesteves.uftm@hotmail.com

Titulação Acadêmica: Graduada em Psicologia

Universidade Federal do Triângulo Mineiro

Afiliação Institucional: Universidade Federal do

Triângulo Mineiro (UFTM)

Nome: Juliana Ruiz Machado.

E-mail: julianamruiz@hotmail.com

Titulação Acadêmica: Mestranda em Psicologia pela

Universidade Federal do Triângulo Mineiro.

Afiliação Institucional: Universidade Federal do

Triângulo Mineiro (UFTM)

\section{Nome: Dennis Gabiatti Lopes.}

E-mail: dennis.gabiatti@gmail.com

Titulação Acadêmica: Graduado em Psicologia pela

Universidade Federal do Triângulo Mineiro.

Afiliação Institucional: Universidade Federal do

Triângulo Mineiro (UFTM)

\section{Nome: José Humberto Curtiço Júnior.}

E-mail: zecurtico@hotmail.com

Titulação Acadêmica: Graduado em Psicologia pela Universidade Federal do Triângulo Mineiro.

Afiliação Institucional: Universidade Federal do

Triângulo Mineiro (UFTM)

\section{Nome: Sabrina Martins Barroso.}

E-mail:smb.uftm@gmail.com

Titulação Acadêmica: Doutora em Saúde

Epidemiológica pela UFMG

Afiliação Institucional: Universidade Federal do

Triângulo Mineiro (UFTM) 